\title{
Acute traumatic thoracolumbar paraspinal compartment syndrome: case report
}

\author{
Katharine D. Harper, MD,, Dayna Phillips, MD, ${ }^{1}$ Joseph M. Lopez, MD, ${ }^{2}$ and Zeeshan Sardar, MD1 \\ Departments of ${ }^{1}$ Orthopaedic Surgery and Sports Medicine and ${ }^{2}$ Trauma and Surgical Critical Care, Temple University Hospital, \\ Philadelphia, Pennsylvania
}

\begin{abstract}
Although compartment syndrome can occur in any compartment in the body, it rarely occurs in the paraspinal musculature and has therefore only been reported in a few case reports. Despite its rare occurrence, acute paraspinal compartment syndrome has been shown to occur secondary to reperfusion injury and traumatic and atraumatic causes. Diagnosis can be based on clinical examination findings, MRI or CT studies, or through direct measurement of intramuscular pressures. Conservative management should only be used in the setting of chronic presentation. Operative decompression via fasciotomy in cases of acute presentation may improve the patient's symptoms and outcomes. When treating acute paraspinal compartment syndrome via surgical decompression, an important aspect is the anatomical consideration. Although grouped under one name, each paraspinal muscle is enclosed within its own fascial compartment, all of which must be addressed to achieve an adequate decompression. The authors present the case of a 43-year-old female patient who presented to the emergency department with increasing low-back and flank pain after a fall. Associated sensory deficits in a cutaneous distribution combined with imaging and clinical findings contributed to the diagnosis of acute traumatic paraspinal compartment syndrome. The authors discuss this case and describe their surgical technique for managing acute paraspinal compartment syndrome.

https://thejns.org/doi/abs/10.3171/2018.6.SPINE18186
\end{abstract}

KEYWORDS paraspinal compartment syndrome; surgical management; trauma; surgical technique

\section{A} CUTE compartment syndrome is a condition in which pressures within a compartment limit the perfusion within that specific compartment. This can result in muscle and nerve ischemia. Untreated or undiagnosed compartment syndrome can lead to muscle necrosis, nerve injuries, wound closure problems, longterm pain, and even death. ${ }^{3}$ Once identified, fasciotomies for the extremities should be performed within 6 hours of presentation and no more than 12 hours after onset. ${ }^{2,13}$ Although this condition can occur in any compartment in the body, it rarely occurs in the paraspinal musculature and therefore is not well studied in this setting. In our review of the literature, we found that $17 \mathrm{pa}-$ tients have been previously identified as having an acute paraspinal compartment syndrome; however, none of the reports addressed the details of surgical management with a technical description. . $^{1,5,5,10,11}$ We describe a case of acute, traumatic paraspinal compartment syndrome and the path leading to its diagnosis and treatment. Surgical fasciotomy led to a positive response without significant neurological deficits postoperatively.

\section{Case Report History and Presentation}

A 43-year-old woman with no significant spine-related history presented to the emergency department, describing low-back and flank pain that had been present for less than a day. The pain initially began after she fell several feet off her bed and hit her lower back on a step stool. Afterwards she was unable to ambulate due to pain. Her symptoms rapidly progressed, leading to her presentation in the emergency department. She rated her back pain as a 10 of 10 on the visual analog scale and described it as excruciating. Although present throughout her entire middle and low back, the pain was worse on the left side.

\section{Examination}

On physical examination, she was found to have tenderness to palpation in the T10-L3 paraspinal region bilaterally. Body habitus of the patient made it difficult to assess for swelling (BMI $45.2 \mathrm{~kg} / \mathrm{m}^{2}$ ). Her left paraspinal tissues were found to be firm and noncompressible, as

ABBREVIATIONS CK = creatinine kinase; ICU = intensive care unit. 
well as exquisitely tender. She had a sensation deficit to light touch on the left flank and left lower back roughly from the T10 to L3 level from the middle of her back to the midaxillary line. She had normal sensory and motor function of her lower extremities bilaterally.

\section{Investigations Performed}

The differential diagnosis at this point included traumatic injury to the thoracolumbar spine, renal or retroperitoneal pathology due to the flank pain, and soft-tissue hematoma. A urine specimen and blood chemistry studies were sent upon initial presentation. Testing revealed a serum creatinine kinase $(\mathrm{CK})$ of $86,174 \mathrm{U} / \mathrm{L}$ (reference range 38-140 U/L) and 4+ blood on urinalysis. Blood urea nitrogen was $13 \mathrm{mg} / \mathrm{dl}$, creatinine of $1.23 \mathrm{mg} / \mathrm{dl}$, hemoglobin of $12.2 \mathrm{~g} / \mathrm{dl}$, and white blood cell count of $14.8 \mathrm{~K} / \mathrm{mm}^{3}$. All remaining studies were within normal limits. An abdominal CT contrast-enhanced study showed enlargement of the left paraspinal musculature compartment (Fig. 1). No abnormalities were seen related to the kidneys, retroperitoneum, bowel, or vasculature.

Due to the patient's history of trauma, pain out of proportion that was unresponsive to medications, and clinical, laboratory, and imaging findings, paraspinal compartment syndrome was suspected. Compartment pressures were measured with the patient in a lateral position using a Compartment Pressure Monitoring Kit (Centurion Medical Products). Pressures were measured in the paraspinal musculature at approximately the L2 level, the level the patient reported the most pain on palpation. Right and left paraspinal musculature pressures measured 13 and $21 \mathrm{~mm}$ $\mathrm{Hg}$, respectively.

\section{Surgical Technique}

The patient was taken emergently to the operating room for thoracolumbar paraspinal fasciotomies. Surgical incision was performed about 7 hours from the time of the patient's initial presentation to the emergency department. A midline incision directly over the T10-L4 spinous processes was performed and dissection was carried to the thoracolumbar fascia. Following this, a plane was dissected out between the thoracolumbar fascia and the individual tendinous aponeurosis layers of the paraspinal musculature (Fig. 2A). Once this plane was developed, decompression of the individual muscle fascias was performed. The multifidus was decompressed by releasing the muscle bilaterally along the spinous processes to the junction of the spinous process and lamina (Fig. 2B). The longissimus and iliocostalis muscles were each decompressed bilaterally by making a longitudinal incision in their tendinous aponeuroses, respectively. Upon inspection of the left-sided musculature, from T12 to L3, there was reduced contractility, as well as pale-appearing muscle belly without evidence of necrosis. Findings on the right side revealed healthier, bleeding, contractile muscle.

Following the release, a skin-only closure was performed with drain placements to avoid seroma development in the opened fascial plane (Fig. 2C). Should there be considerable tension on the skin during closure, consideration should be given to placing a vacuum-assisted clo-

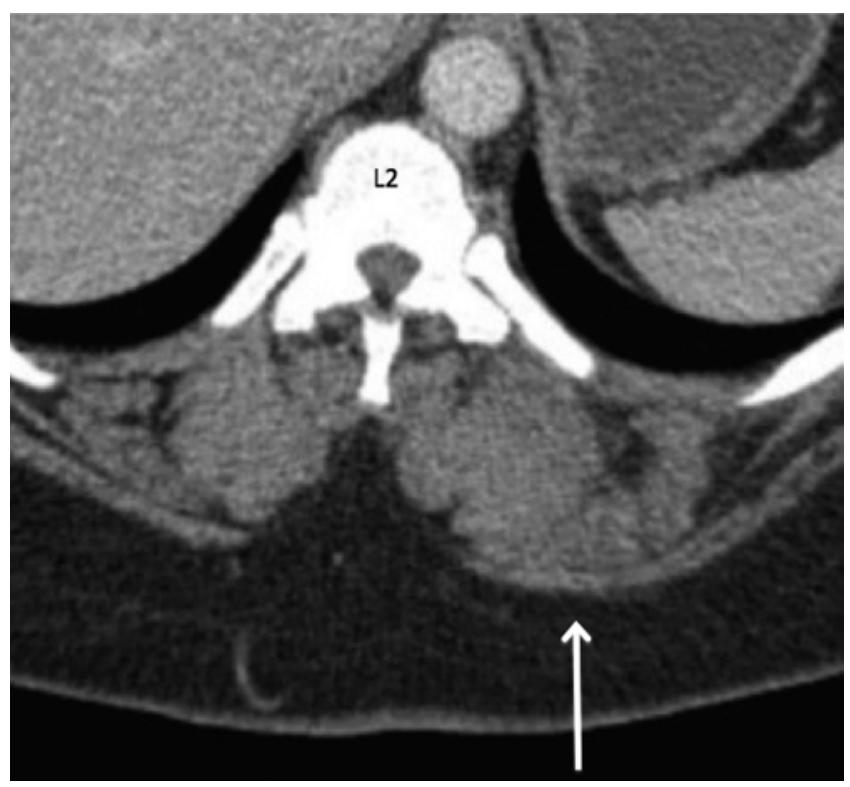

FIG. 1. CT scan at the T12 level demonstrating left-sided paraspinal musculature swelling (white arrow).

sure device and delaying closure for 3-5 days. If there is concern regarding the amount of muscle necrosis found at initial presentation and decompression, a vacuum-assisted closure device should be placed and a repeat debridement scheduled in 2-3 days.

After the surgery, the patient was taken to the surgical intensive care unit (ICU).

\section{Postoperative Course}

During a postoperative evaluation 4 hours after surgery, the patient stated she felt significant improvement in both her pain and her flank sensation, although a deficit was still present. Throughout her 6-day hospital stay, the patient's back pain and sensation continued to improve, with only a deficit remaining directly over the left paraspinal muscle. CK levels were followed throughout her stay and showed a downward trend from 86,174 $\mathrm{U} / \mathrm{L}$ at presentation to $36,163 \mathrm{U} / \mathrm{L}$ on postoperative day 1 to $2512 \mathrm{U} / \mathrm{L}$ on day of discharge. By 6 months postoperatively, the patient reported a significant improvement in back pain. She rated her back pain as 5 of 10 on a visual analog scale. At the time of her last follow-up, she had completed her physical therapy course and was experiencing no deficits in her activities of daily living. She has returned to her baseline activity levels.

\section{Discussion}

Paraspinal compartment syndrome can be classified as either acute or chronic. Typical presentation for patients with chronic compartment syndrome is a prolonged history of atraumatic back pain. Patients with acute compartment syndrome will report a shorter duration of symptoms with a possible inciting factor of trauma, exercise, or surgery related. Our patient presented with acute, traumatic paraspinal compartment syndrome as she had suffered 

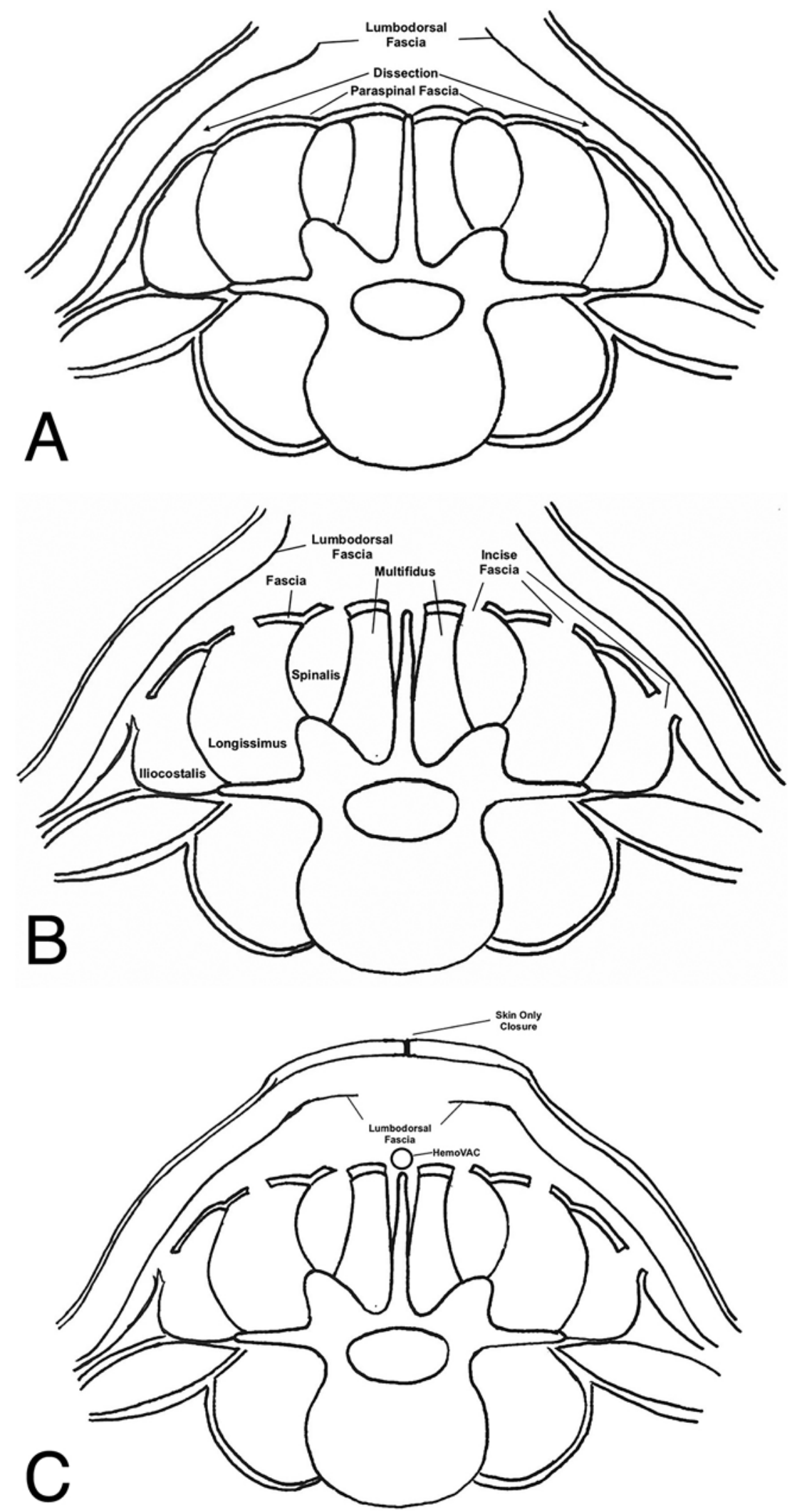

FIG. 2. A: Illustration demonstrating the fascial plane between the thoracolumbar fascia and individual muscle compartment fascias, which require dissection and individual release. B: Illustration demonstrating the location of each individual fascial incision, resulting in the decompression of iliocostalis, spinalis, longissimus, and multifidus muscle bellies. C: Illustration demonstrating a skin-only closure of the back with the thoracolumbar fascia left open and Hemovac drains in place to avoid seroma development. 
a recent traumatic event and had no history of back pain prior to the onset of her acute pain.

In our review of the literature concerning acute paraspinal compartment syndrome, the majority of cases were caused by exercise or had surgery-related causes (i.e., reperfusion injury). ${ }^{1,4,6,8,10,14,15}$ Sava et al. reported the only case of paraspinal compartment syndrome secondary to trauma. Similar to our patient, their case involved a female patient of the same age who had had no spine-related fractures. Although compartment syndrome can occur in association with lower-extremity fractures, no cases have been reported in relation to spinal fractures. In a review performed by Alfaraj et al., the majority of acute paraspinal compartment syndromes were in men and in patients under the age of 40 years. ${ }^{1}$ The review revealed that most cases of paraspinal compartment syndrome were due to exercise, followed by non-spine surgery (such as aortic bypass or gastric bypass), drug abuse, and trauma (direct injury to paraspinal muscles). ${ }^{1}$ Alfaraj et al. also provided a classification for paraspinal compartment syndrome, with type I being acute with 3 subtypes (A, atraumatic; B, direct trauma; and $\mathrm{C}$, non-spine surgery) and type II being chronic exertional. ${ }^{1}$ No specific patient demographics that might influence presentation and response to different treatment options have been identified in previous studies.

In the few studies of paraspinal compartment syndrome, imaging studies such as MRI and CT were performed and provided some aid in the diagnosis. In the case report by Khan et al., both imaging studies were used. Initial CT findings showed paraspinal muscle swelling and paralytic ileus. MRI was later performed and demonstrated increased signal in the paraspinal muscles bilaterally on T2-weighted imaging. ${ }^{4}$ In some cases, MRI was used secondarily to further assess the musculature due to negative CT findings and a patient's continued or worsening symptoms ${ }^{1,8}$ In a review by Nathan et al., MRI was used in several reports as the initial imaging modality. ${ }^{10}$ Our patient had abdominal CT scanning performed as the initial imaging modality due to the flank pain; the study showed the enlargement of paraspinal musculature. An MRI study was determined to be unnecessary as there was swelling evident on CT scan, and we had laboratory and physical examination findings that suggested a diagnosis of paraspinal compartment syndrome. With several reports, including our own, contributing to the literature, no study has determined which modality is best for the initial diagnosis. Further studies should be performed to assess the use of MRI and CT regarding their sensitivity and specificity in diagnosing paraspinal compartment syndrome.

Laboratory values also aid in the diagnosis of paraspinal compartment syndrome by permitting evaluation of secondary conditions such as rhabdomyolysis and renal impairment. Laboratory tests such as those for creatinine phosphokinase, basic metabolic panel, urinalysis, and serum and urine myoglobin were performed in the majority of case reports. ${ }^{1,410,14}$ Treatment should begin as soon as the diagnosis of paraspinal compartment syndrome is established, especially in the setting of rhabdomyolysis. In the case of our patient, $\mathrm{CK}$, basic metabolic panel, and urinalysis studies were completed. In order to preserve re- nal function, intravenous fluids were administered prior to surgical decompression. ICU admission following decompression for rhabdomyolysis prevention or management should be considered. Due to our patient's CK values, she was admitted to ICU for close monitoring and fluid management.

In patients presenting with acute back pain, other etiologies besides paraspinal compartment syndrome must be considered and ruled out. What must also be considered are findings specific to paraspinal compartment syndrome that may aid in differentiating it from other causes. This includes disc herniation, retroperitoneal pathology, and fractures. Our patient presented with tenderness to palpation over paraspinal musculature, firm, noncompressible paraspinal muscles, and decreased sensation in the paraspinal region to the midaxillary line. Similar findings, such as decreased or absent bowel sounds, have been observed in other cases. ${ }^{4,10}$ MRI and CT studies used to aid in the diagnosis of paraspinal compartment syndrome can also be obtained to rule out other etiologies. Most importantly, the patient's history pertaining to any trauma that may have caused the pain, activities performed prior to or when the pain began, and a history of similar back pain should also be assessed. Time from diagnosis to surgical decompression is of great importance. In the case of our patient, it took 6 hours to make the diagnosis of paraspinal compartment syndrome and surgery was performed 1 hour later.

The anatomy and position of the patient come into play when measuring compartment pressures, no different than extremity measurements. When measuring compartments in the extremity, the monitor should be held parallel to the ground and calibrated just prior to insertion into the compartment. The extremity being measured should not be resting on the bed, as this can artificially increase the pressure measurement, and it, too, should be in a position parallel to the ground. When measuring pressures in the paraspinal compartments, the same principles mentioned above apply, especially those pertaining to positioning. Unlike in the extremities, where a value within $30 \mathrm{~mm}$ $\mathrm{Hg}$ of diastolic blood pressure ${ }^{9}$ or an absolute value of 45 $\mathrm{mm} \mathrm{Hg}^{7}$ (reference values of $8 \mathrm{~mm} \mathrm{Hg}{ }^{12}$ ) is considered diagnostic of compartment syndrome, control values for paraspinal compartment pressures have not been well established. Anatomical studies assessing the different pressures measured within the paraspinal muscle compartments have shown large variability in values depending on the patient's position. Songcharoen et al. measured baseline compartment pressures in varying positions. The resting intramuscular pressure was found to be $3.11 \mathrm{~mm}$ $\mathrm{Hg}$ while in a prone position, $4.47 \mathrm{~mm} \mathrm{Hg}$ while standing, and $7.95 \mathrm{~mm} \mathrm{Hg}$ while sitting..$^{15}$ Previous studies have shown a diagnosis of compartment syndrome with measurements anywhere from $26 \mathrm{~mm} \mathrm{Hg}^{4}$ to $108 \mathrm{~mm} \mathrm{Hg}{ }^{8}$ This shows the wide ranges of pressures at which patients may become symptomatic, and no study had identified patients at risk at lower values. Figure 3 presents an algorithm for diagnosis of suspected paraspinal compartment syndrome, whereas Table 1 presents the investigational findings potentially associated with paraspinal compartment syndrome. 


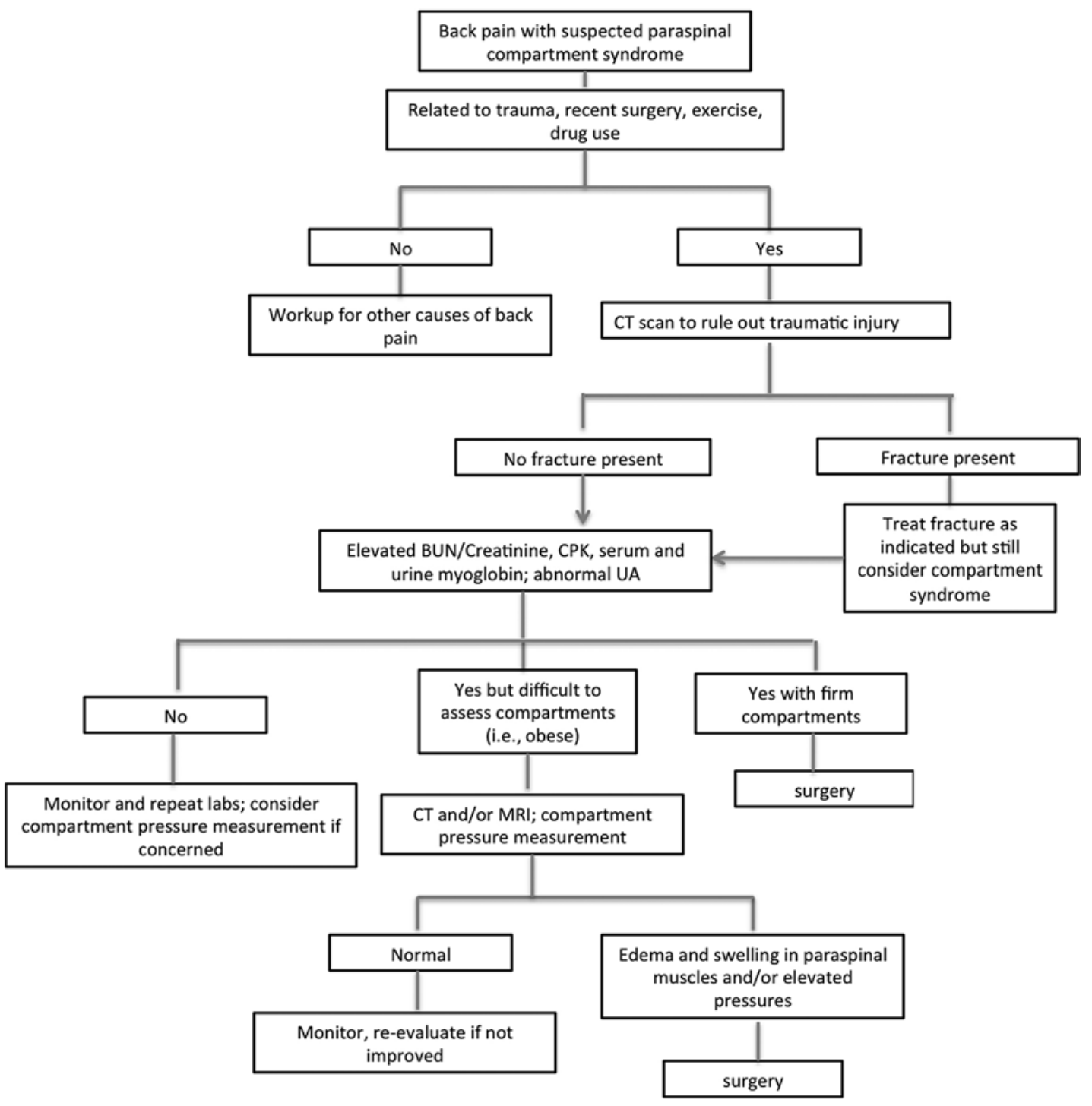

FIG. 3. A treatment algorithm for paraspinal compartment syndrome, including investigations at presentation. In all situations, a high suspicion for compartment syndrome should be maintained with frequent monitoring. Once a diagnosis is confirmed, proceeding to surgery may be the appropriate choice. $\mathrm{BUN}=$ blood urea nitrogen; $\mathrm{CPK}=$ creatinine phosphokinase; $\mathrm{UA}=$ urinalysis.

TABLE 1. Parameters for the diagnosis of paraspinal compartment syndrome

\begin{tabular}{ll}
\hline \multicolumn{1}{c}{ Investigation } & \multicolumn{1}{c}{ Findings in Paraspinal Compartment Syndrome } \\
\hline Blood urea nitrogen & Normal or elevated (reference range $8-20 \mathrm{mg} / \mathrm{dl}$ ) \\
\hline Creatinine & Normal or elevated (reference range $0.60-1.10 \mathrm{mg} / \mathrm{dl}$ ) \\
\hline CK & Elevated $>1000$ (reference range $38-140 \mathrm{U} / \mathrm{L}$ ) \\
\hline Serum myoglobin & Elevated (reference range $0-0.003 \mathrm{mg} / \mathrm{dl}$ ) \\
\hline Urine myoglobin & If present, suggestive of rhabdomyolysis (normal is absence of myoglobin in urine) \\
\hline Urinalysis & Positive for blood (normal is absence of blood in urine) \\
\hline Paraspinal compartment pressures & Elevated but highly variable depending on position (normal $<8 \mathrm{~mm} \mathrm{Hg}$ ) \\
\hline Lumbar spine MRI & Increased signal in paraspinal musculature on T2-weighted images \\
\hline Lumbar spine or abdomen CT & Enlargement of paraspinal musculature \\
\hline
\end{tabular}

Lists the investigational modalities and associated findings used to diagnose paraspinal compartment syndrome. 
Nonoperative and operative management of paraspinal compartment syndrome can be used with varying degrees of success. Conservative management has included intravenous fluids, pain control, bed rest, and monitoring of kidney function., ${ }^{1,410}$ When the diagnosis is confirmed, operative management via surgical decompression is considered to be the optimal form of treatment. Multiple reports have shown ongoing long-term sequelae in patients managed conservatively when presenting with exertional paraspinal compartment syndrome, and the long-term outcomes have improved once surgical decompression has been performed. ${ }^{4,5,8,11}$ While many studies exist that have established the optimal timing for extremity decompression in cases of acute compartment syndrome (ideally within 6 hours, with no more than 12 hours to ensure minimal muscle necrosis), ${ }^{2,13}$ no studies have evaluated the optimal timing of surgical decompression to avoid longterm sequelae in the paraspinal muscles. We recommend that in patients presenting with acute, rapidly progressing muscular back pain, acute paraspinal compartment syndrome must be considered and surgical treatment offered if it is diagnosed.

\section{References}

1. Alfaraj AM, Alfaraj ZM, Alsahwan AG: Acute lumbar paraspinal compartment syndrome: a case report and detailed literature review. Int Surg J 4:775-779, 2017

2. Finkelstein JA, Hunter GA, Hu RW: Lower limb compartment syndrome: course after delayed fasciotomy. J Trauma 40:342-344, 1996

3. Kashuk JL, Moore EE, Pinski S, Johnson JL, Moore JB, Morgan S, et al: Lower extremity compartment syndrome in the acute care surgery paradigm: safety lessons learned. Patient Saf Surg 3:11, 2009

4. Khan RJ, Fick DP, Guier CA, Menolascino MJ, Neal MC: Acute paraspinal compartment syndrome. A case report. J Bone Joint Surg Am 87:1126-1128, 2005

5. Kitajima I, Tachibana S, Hirota Y, Nakamichi K: Acute paraspinal muscle compartment syndrome treated with surgical decompression: a case report. Am J Sports Med 30:283285,2002

6. Konno S, Kikuchi S, Nagaosa Y: The relationship between intramuscular pressure of the paraspinal muscles and low back pain. Spine (Phila Pa 1976) 19:2186-2189, 1994

7. Matsen FA III, Winquist RA, Krugmire RB Jr: Diagnosis and management of compartmental syndromes. J Bone Joint Surg Am 62:286-291, 1980
8. Minnema BJ, Neligan PC, Quraishi NA, Fehlings MG, Prakash S: A case of occult compartment syndrome and nonresolving rhabdomyolysis. J Gen Intern Med 23:871-874, 2008

9. Mubarak SJ, Owen CA, Hargens AR, Garetto LP, Akeson WH: Acute compartment syndromes: diagnosis and treatment with the aid of the wick catheter. J Bone Joint Surg Am 60:1091-1095, 1978

10. Nathan ST, Roberts CS, Deliberato D: Lumbar paraspinal compartment syndrome. Int Orthop 36:1221-1227, 2012

11. Osamura N, Takahashi K, Endo M, Kurumaya H, Shima I: Lumbar paraspinal myonecrosis after abdominal vascular surgery: a case report. Spine (Phila Pa 1976) 25:1852-1854, 2000

12. Raza H, Mahapatra A: Acute compartment syndrome in orthopedics: causes, diagnosis, and management. Adv Orthop 2015:543412, 2015

13. Ritenour AE, Dorlac WC, Fang R, Woods T, Jenkins DH, Flaherty SF, et al: Complications after fasciotomy revision and delayed compartment release in combat patients. J Trauma 64 (2 Suppl):S153-S162, 2008

14. Sava J, Moelleken A, Waxman K: Cardiac arrest caused by reperfusion injury after lumbar paraspinal compartment syndrome. J Trauma 46:196-197, 1999

15. Songcharoen $P$, Chotigavanich $C$, Thanapipatsiri S: Lumbar paraspinal compartment pressure in back muscle exercise. J Spinal Disord 7:49-53, 1994

\section{Disclosures}

The authors report no conflict of interest concerning the materials or methods used in this study or the findings specified in this paper.

\section{Author Contributions}

Conception and design: all authors. Acquisition of data: all authors. Analysis and interpretation of data: Harper, Phillips, Sardar. Drafting the article: Harper, Phillips. Critically revising the article: Harper, Sardar. Reviewed submitted version of manuscript: all authors. Approved the final version of the manuscript on behalf of all authors: Harper. Study supervision: Sardar.

\section{Correspondence}

Katharine D. Harper: Temple University Hospital, Philadelphia, PA. katharineharpermd@gmail.com. 